\title{
INSIDE THE USCAP JOURNALS
}

\section{MODERN PATHOLOGY}

\section{The relationship between soft-tissue aneurysmal bone cysts and MO https://doi.org/10.1038/s41379-020-0513-4}
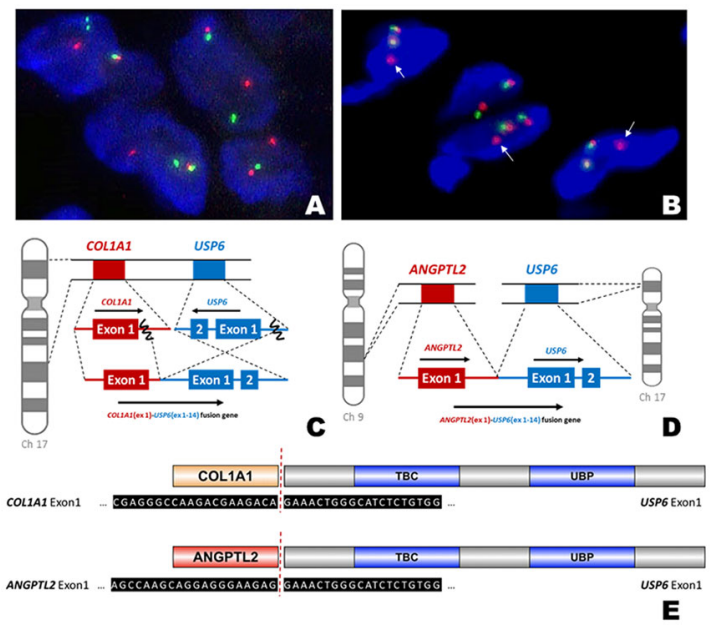

Zhang et al. investigated the relationship between characteristics of soft-tissue aneurysmal bone cysts (STABCs) and myositis ossificans (MO) using imaging as well as fluorescence in situ hybridization (FISH) for USP6 rearrangement. Lesions were shown to be frequently associated with peripheral calcification, cystic components, and perilesional edema with USP6 rearrangement in all seven cases. COL1A1-USP6 was identified through RNA-seq as a fusion in six of seven cases, and a novel ANGPTL2-USP6 fusion was identified in one case. En bloc excision is curative when the lesions cause symptoms, and the patients have shown continued disease-free survival at follow-ups. Given the frequency of similarities in clinical, radiological, and pathological outcomes across the patients with either STABC or $\mathrm{MO}$, the group concludes that the two conditions are closely related. Further investigation is needed into the functional implications of the novel fusion of ANGPTL2-USP6.

\section{Exploring the pathogenesis of primary mammary angiosarcoma https://doi.org/10.1038/s41379-020-0511-6}
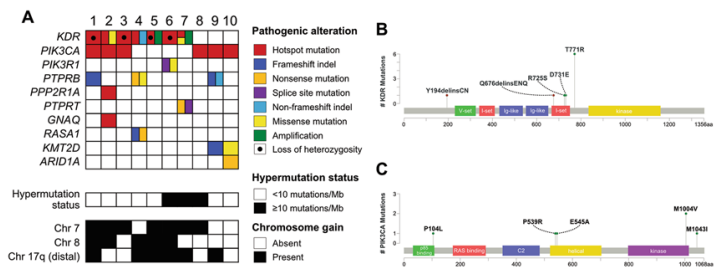

Primary angiosarcoma (AS) accounts for only $<0.05 \%$ of breast malignancies; secondary mammary AS following radiation therapy is more common. Beca et al. sought to distinguish between primary mammary AS (PMAS) and secondary AS. To further characterize the pathogenesis of PMAS, the group assessed 10 cases and found recurrent genomic alterations in KDR (VEGFR2), PIK3CA/PIK3R1, and PTPRB at higher frequencies than reported in AS across all sites. Six of 10 tumors contained a KDRp.T771R hotspot mutation, and six of seven cases showed pathogenic PI3K mutations; every tumor in the set held a mutation in one and/or the other. The group proposes that further investigation will reveal more distinguishable features of PMAS, which could influence therapeutic mechanisms in the future.

\section{LABORATORY INVESTIGATION}

\section{Analysis of the tumor microenvironment using MIBI} https://doi.org/10.1038/s41374-020-0417-4

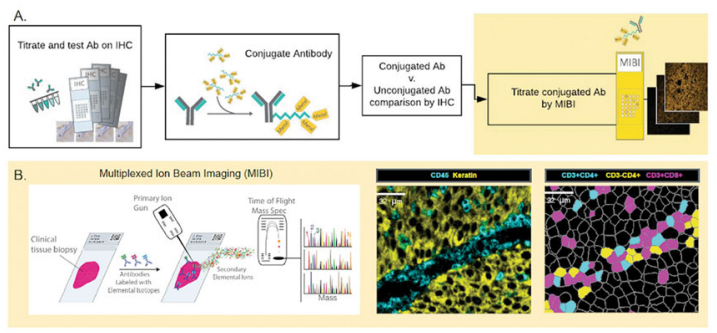

Ptacek et al. sought to demonstrate the applicability of multiplexed ion beam imaging (MIBI) to characterizing the cellular composition and spatial organization of the tumor microenvironment (TME), which has been largely difficult to achieve using multiplexed immunohistochemistry.

Formalin-fixed paraffin-embedded samples were simultaneously stained with 15 metal isotopelabeled-antibodies and processed across the TME and down to single cells. Frequencies of different cell subsets and the distributions of nearest-neighbor distances between them were calculated using these data. Infiltrating cytotoxic T cells and macrophages were observed, to differing degrees, between samples. Details of the spatial arrangement and relationship between the cells could be seen when looking at admixed PD-L1 ${ }^{+}$ 
macrophages and $\mathrm{PD} 1^{+} \mathrm{T}$ cells. Spatial relationships within the TME have been shown to be associated with overall survival in other studies, so the team proposes that this technique will provide additional insights into the drivers of immune dysfunction in the TME.

\section{The role of PAR1-TRPV4 in vascular hyperpermeability} https://doi.org/10.1038/s41374-0200430-7

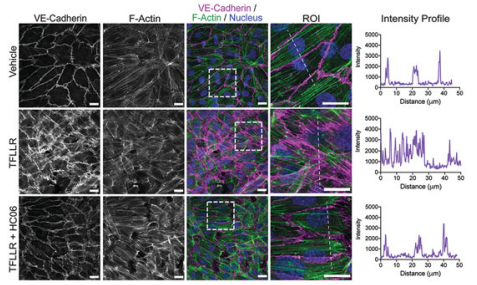

While roles of protease-activated receptor 2 (PAR2) and the ion channel transient receptor potential vanilloid 4 (TRPV4) have been investigated, Peng et al. examinedin the context of vascular permeabilitytheir roles specifically in the control of edema mediated by thrombin-induced signaling. TRPV4 contributed to PAR1induced vascular hyperpermeability in the airways and upper gastrointestinal tract of mice by contributing to sustained PAR1induced $\mathrm{Ca}^{2+}$ signaling, as demonstrated using Evans Blue permeation and retention as an indicator. Because TRPV4 is a signaling effector for several PAR family members, and inhibition of TRPV4 diminishes PAR1-induced hyperpermeability, the group proposes that TRPV4 may also contribute to other PAR1-mediated signaling processes. Further investigation is needed to fully assess the therapeutic impact and benefits of these findings.

\section{nature.com/pathology Interplay of immune infiltration and genomic features modulates \\ response to PD-1 blockade}

By analyzing 592 samples of clear-cell renal cell carcinoma, Braun et al. investigated the drivers and resistors of PD-1 blockade in these tumors and uncover the immunogenomic determinants of the therapeutic response. Consistently, neither $\mathrm{CD}^{+}$T-cell infiltration nor somatic alteration burden and HLA zygosity were associated with clinical response. Among tumors that exhibited this infiltration, however, a depletion in PBRM1 mutations was observed, along with enrichment for chromosomal losses of 9p21.3. The group demonstrated the interaction of immune infiltration and somatic alterations in the context of anti-PD-1-based combination therapies. These data, while in need of additional

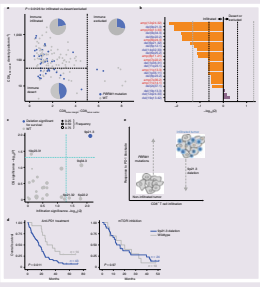
validation through clinical trials and increased sample numbers, could influence the design of those trials and one day impact the selection of patients most likely to respond preferably to PD-1/PD-L1 blockade as a therapeutic regime. Nature Medicine 2020;26:909-918; https://doi.org/10.1038/s41591-020-0839-y

\section{Mutational impact of colibactin DNA damage in colorectal cancer}

Given the frequency of cancers that originate in the mucosal epithelium, a tissue commonly damaged by chronic bacterial infections, Dziubańska-Kusibab sought to identify DNA-damage signatures that might be present in colibactin-infected human colorectal cells. Colibactin-induced DNA double-strand breaks (DSBs) were enriched for AT-rich hexameric sequence motifs, with specific DNA-shape characteristics. Notable enrichment of these motifs was shown in colorectal cancers when a database of somatic mutations was assessed with exact DSB loci, next to the alkylated adenines, corresponding to mutational hot spots in cancer genomes. The data showed that DSB cleavage occurs immediately downstream $\left(5^{\prime}>3^{\prime}\right)$ to positions 2 and 5 of the

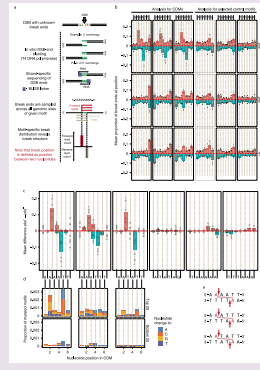
putatively alkylated adenines on the opposing strands, with cleavage resulting in staggered ends with $5^{\prime}$ overhangs of two nucleotides each. The data showed colibactin-related driver mutations in colorectal cancer, suggesting a potential causal relationship that has yet to be explored for usefulness in therapeutics. Nature Medicine, published online 1 June 2020; https://doi.org/10.1038/s41591-020-0908-2

\section{Deep learning for the differential diagnosis of skin conditions}

Owing to a shortage of dermatologists, diagnosis of skin diseases often falls to general practitioners, resulting in lower diagnostic accuracy. Liu et al. developed a deep learning system (DLS) to analyze 16,114 de-identified cases (photographs and clinical data) in order to provide a differential diagnosis of skin diseases. Twenty-six common skin conditions were differentiated in the system, with an additional 419 secondary predictions. Three board-certified dermatologists

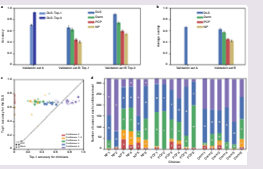
validated 963 cases. The DLS's accuracy was non-inferior to that of six other dermatologists and superior to that of six primary care physicians and six nurse practitioners. Increasing the quantity of contextual images (metadata) in the DLS for each case improved accuracy another 4-5\%. The authors propose that the DLS can assist general practitioners in diagnosing skin conditions through storing-andforwarding teledermatology, which will assisting with triaging, reduce wait times for specialty care, and enable earlier initiation of treatment.

Nature Medicine 2020; https://doi.org/10.1038/s41591-020-0842-3 